\title{
Novel acridone-modified MCM-41 type silica: Synthesis, characterization and fluorescence tuning
}

\author{
Maximilian Hemgesberg ${ }^{1}$, Gunder Dörr ${ }^{1}$, Yvonne Schmitt ${ }^{1}$, Andreas Seifert ${ }^{2}$, \\ Zhou Zhou ${ }^{3}$, Robin Klupp Taylor ${ }^{4}$, Sarah Bay ${ }^{5}$, Stefan Ernst ${ }^{1}$, \\ Markus Gerhards ${ }^{1}$, Thomas J. J. Müller ${ }^{5}$ and Werner R. Thiel ${ }^{* 1}$
}

\section{Full Research Paper}

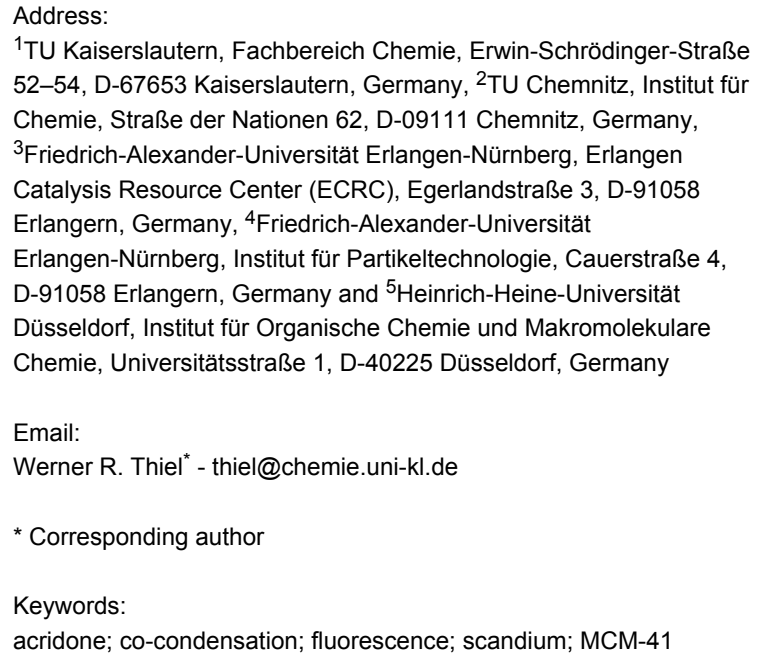

${ }^{1} \mathrm{TU}$ Kaiserslautern, Fachbereich Chemie, Erwin-Schrödinger-Straße 52-54, D-67653 Kaiserslautern, Germany, ${ }^{2} \mathrm{TU}$ Chemnitz, Institut für Chemie, Straße der Nationen 62, D-09111 Chemnitz, Germany, ${ }^{3}$ Friedrich-Alexander-Universität Erlangen-Nürnberg, Erlangen Catalysis Resource Center (ECRC), Egerlandstraße 3, D-91058 Erlangern, Germany, ${ }^{4}$ Friedrich-Alexander-Universität Erlangen-Nürnberg, Institut für Partikeltechnologie, Cauerstraße 4, D-91058 Erlangern, Germany and ${ }^{5}$ Heinrich-Heine-Universität Düsseldorf, Institut für Organische Chemie und Makromolekulare Chemie, Universitätsstraße 1, D-40225 Düsseldorf, Germany

Email:

Werner R. Thiel ${ }^{*}$ - thiel@chemie.uni-kl.de

* Corresponding author

Keywords:

acridone; co-condensation; fluorescence; scandium; MCM-41

Open Access

Beilstein J. Nanotechnol. 2011, 2, 284-292.

doi:10.3762/bjnano. 2.33

Received: 21 March 2011

Accepted: 12 May 2011

Published: 09 June 2011

This article is part of the Thematic Series "Micro- and mesoporous solids: From science to application"

Guest Editor: J. J. Schneider

(c) 2011 Hemgesberg et al; licensee Beilstein-Institut. License and terms: see end of document.

\begin{abstract}
A Mobil Composition of Matter (MCM)-41 type mesoporous silica material containing $N$-propylacridone groups has been successfully prepared by co-condensation of an appropriate organic precursor with tetraethyl orthosilicate (TEOS) under alkaline sol-gel conditions. The resulting material was fully characterized by means of X-ray diffraction (XRD), $\mathrm{N}_{2}$-adsorption-desorption, transmission electron microscopy (TEM), IR and UV-vis spectroscopy, as well as ${ }^{29} \mathrm{Si}$ and ${ }^{13} \mathrm{C}$ CP-MAS NMR techniques. The material features a high inner surface area and a highly ordered two-dimensional hexagonal pore structure. The fluorescence properties of the organic chromophore can be tuned via complexation of its carbonyl group with scandium triflate, which makes the material a good candidate for solid state sensors and optics. The successful synthesis of highly ordered MCM materials through co-condensation was found to be dependent on the chemical interaction of the different precursors.
\end{abstract}

\section{Introduction}

Mesoporous silicates are widely used for a variety of applications such as gas storage and heterogeneous catalysis, e.g., the synthesis of $\varepsilon$-caprolactam [1], or the decomposition of nitrous oxides [2]. MCM-41, MCM-48 and other silica materials can normally be functionalized either by in situ post-sol-gel modification or by direct co-condensation of different types of organic 
precursors [3]. The latter method often leads to a more homogeneous distribution of the desired functionalization within the material. It also provides the possibility to characterize application-tailored sol-gel precursors prior to implementing them into the solid, thus increasing the depth of information compared to the data solely drawn from solid state measurements.

Focusing on the synthesis of novel inorganic-organic hybrid materials, we also investigated new ways to produce trialkoxysilanes bearing polycyclic aromatic compounds as terminal groups, $\mathrm{Ar}-\left(\mathrm{CH}_{2}\right)_{n}-\mathrm{Si}(\mathrm{OR})_{3}(n=3, \mathrm{R}=\mathrm{Me}, \mathrm{Et})$, which may lead to interesting optical or electronic properties [4-6] Acridone, being a well-known fluorophore used, e.g., for chemosensors [7], has previously been reported to be suitable for the $\mathrm{p} K_{\mathrm{a}}$ determination of lanthanide salts in aqueous solution, the fluorescence undergoing a bathochromic shift directly correlated to the acidity of the cation bound to its carbonyl group [8]. We therefore sought to create a micro- or mesoporous material containing covalently bound acridone units that would serve the same purpose, by choosing the amino function of acridone as the functional group to be modified. Trialkoxysilanes with a variety of functional groups have already been prepared by $\mathrm{N}$-alkylation of amines using 3-iodopropyltrimethoxysilane (IPTMS) [9] or 3-bromopropyltrimethoxysilane (BPTMS) [10], by sulfamidation [11], by imide [12] or via imine forming reactions [13] using 3-aminopropyltriethoxysilane (APTES). In 2009, an $\mathrm{N}$-alkylated acridone derivative bearing a (tri-isopropyloxysilyl)propyl group and its application as an anion-selective fluorescent probe were reported by Lin and Chen [14]. However, to the best of our knowledge, up to now, no MCM-like material featuring covalently bound acridone units has been described.

\section{Results and Discussion}

Compound 1 was prepared in a two-step sequence (Scheme 1): Following a published procedure, acridone was obtained from commercially available $\mathrm{N}$-phenylanthranilic acid by an acid catalyzed ring closure reaction [15]. To attach the silyl functionalized linker, acridone was deprotonated by $\mathrm{NaH}$ and the resulting anion reacted with 3-iodopropyltrimethoxysilane (IPTMS).
Co-condensation of TEOS with organosilanes in the presence of an appropriate structure-directing template allows the direct and homogeneous incorporation of organic functionalities into a mesoporous material. Applying this method, we were recently able to introduce up to $30 \mathrm{wt} \%$ of redox-active phenothiazines into mesostructured silicas [4-6]. The resulting materials showed a continuous decrease of ordering with increasing bulk of the organic groups, although all exhibited very high specific surface areas. As the formation of micelles will strongly depend on the nature of the organic molecule and its concentration, it was decided to keep the amount of precursor 1 at $10 \mathrm{~mol} \%$ with respect to TEOS in order to be sure that a highly ordered material would be obtained. The MCM-41 analogue was prepared by a modified synthesis previously reported by Pang et al. [15], in which aqueous ethylamine is used in order to adjust smoothly the $\mathrm{pH}$-value of the solution. Given the fact that polycyclic compounds are rather bulky organic moieties which might behave differently when undergoing a sol-gel transformation, a slightly larger molecule (stearyltrimethylammonium bromide, $\mathrm{C}_{18} \mathrm{TAB}$ ) was chosen as the templating agent.

However, obtaining materials with high surface areas has previously been shown to be also dependent on the boiling point of the sol-gel solution. Thus, the addition of larger amounts of volatile organic solvents such as THF to the aqueous phase often prevents the formation of highly ordered mesoporous structures [16]. On the other hand, the $\pi$-stacking of large aromatics generally causes them to be less readily dissolved and requires larger amounts of solvents, which are mostly immiscible with water. Therefore, pure TEOS was tested as a mediating agent for introduction into the sol-gel process. It turned out to be beneficial for the co-condensation process that precursor 1 could be mixed with TEOS rather easily, although it did not prove possible to obtain a homogeneous solution. Despite the fact that it was rather difficult to handle by syringe, a mixture of TEOS and $\mathbf{1}$ could be converted to the corresponding mesostructured silica MCM-ACR.

In contrast to $\mathbf{1}$, a similar compound prepared from pyrenesulfonyl chloride and APTES via sulfamidation immediately yielded a flocculent precipitate when brought in contact with

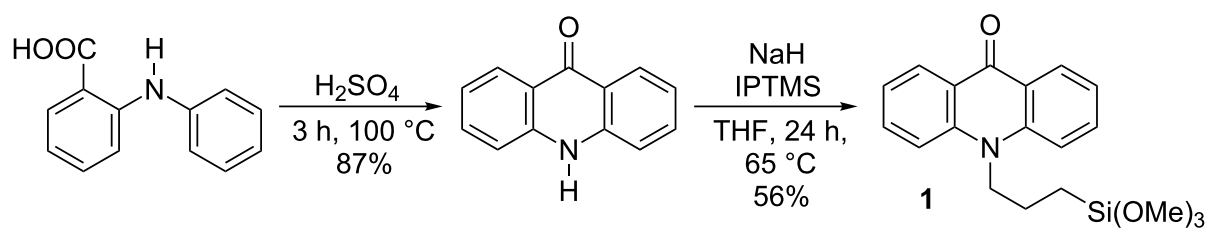


TEOS. This observation might be explained by the combination of the pyrene moieties, forming strong $\pi$-bonding interactions, and the highly polar hydrogen bonding sulfonamide, causing the compound to become inhomogeneous in TEOS. As expected, the material obtained from the pyrene precursor showed a drastically decreased inner surface area of only $322 \mathrm{~m}^{2} \cdot \mathrm{g}^{-1}$ and no ordered material structure at all.

The CHNS analysis of MCM-ACR clearly indicates a slightly increased ratio between the dye and silica (1:7.5) compared to the initial ratio of the synthesis (1:9), probably resulting from different rates of hydrolysis of TEOS and the $\mathrm{RSi}(\mathrm{OMe})_{3}$ groups. Converting the materials' molecular composition to the amount of $N$-propylacridone moieties per gram, we obtained a dye loading of approximately $240 \mathrm{mg} \cdot \mathrm{g}^{-1}$.

From the infrared spectra of $\mathbf{1}$ and both the unmodified as well as the modified MCM-ACR, the aromatic and aliphatic $\mathrm{C}-\mathrm{H}$ vibrational bands around $3000 \mathrm{~cm}^{-1}$ showed reduced intensities in the solid and were mostly covered by the weak, yet broad, absorption bands of the silanol groups and bands from water trapped in the silica framework (Figure 1).

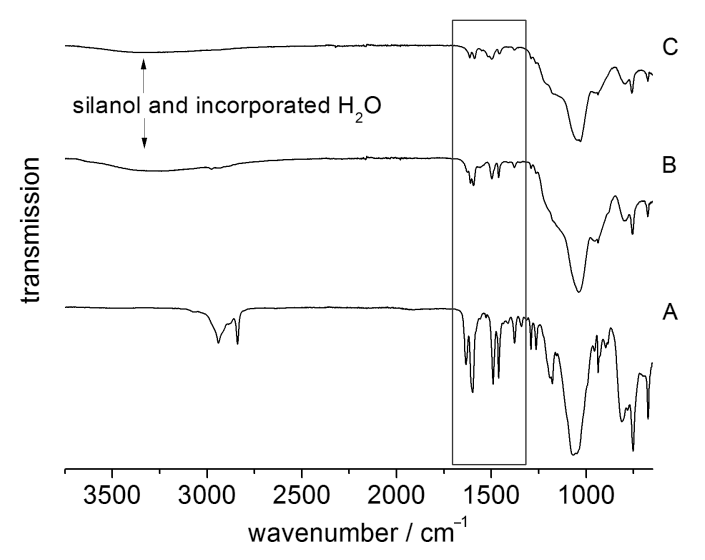

Figure 1: Infrared spectra of compound $1(A)$, MCM-ACR $(B)$ and MCM-ACR + Sc(OTf $)_{3}(C)$. The box marks the section given in Figure 2 (resolution $\pm 2 \mathrm{~cm}^{-1}$ ).

The spectra were also found to be in accordance with the interpretation of the infrared spectrum of free acridone reported earlier by Berezin et al. [17]. By comparing the region between $\tilde{v}=1700 \mathrm{~cm}^{-1}$ and $1300 \mathrm{~cm}^{-1}$, complex formation with the scandium(III) cation is clearly observed. The infrared absorption of the $\mathrm{C}=\mathrm{O}$ vibrational band (precursor 1: $1630 \mathrm{~cm}^{-1}$, $N$-methylacridone: $1630 \mathrm{~cm}^{-1}$ [18]) shifts slightly to lower wavenumbers (Figure 2) after the immobilization, and also splits into two resonances (MCM-ACR: $1625 \mathrm{~cm}^{-1}$, $1609 \mathrm{~cm}^{-1}$ ) indicating an interaction of the carbonyl group with
Lewis or Brønsted acidic or with hydrogen-bond-donating surface sites, which has previously been described for fluorescent probes such as Michler's ketone [19]. This again changes after the reaction with $\mathrm{Sc}(\mathrm{OTf})_{3}$ : A single $\mathrm{C}=\mathrm{O}$ absorption emerges at $1613 \mathrm{~cm}^{-1}$. The redshift of the absorption frequency is conclusive with respect to the weakened $\mathrm{C}=\mathrm{O}$ double bond caused by the electron donation from the carbonyl unit to the scandium(III) cation, which is also evident in the UV-vis absorption of MCM-ACR (see below in Figure 9).

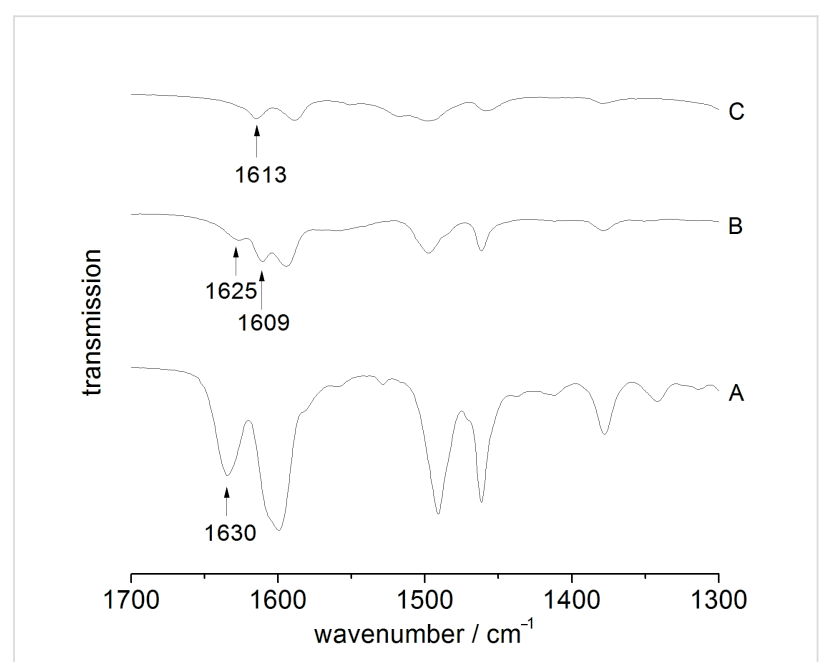

Figure 2: $\mathrm{C}=\mathrm{O}$ vibrational band section of the infrared spectra of compound $1(A)$, MCM-ACR $(B)$ and MCM-ACR + Sc(OTf $)_{3}(C)$.

According to the TEM analysis (Figure 3) and BET measurements (Figure 4), the sol-gel process yielded a well ordered mesoporous material with a total surface area of up to $810 \mathrm{~m}^{2} \cdot \mathrm{g}^{-1}$, exhibiting a characteristic pore size distribution with a sharp peak around $2.4 \mathrm{~nm}$. The XRD spectrum reveals the expected peak patterns only for the (110) and (200) Miller indices as the (100) peak is not distinguishable from the primary beam (Figure 5). BET data of an authentic MCM-41 sample previously prepared in our group corresponded to a total surface area of $1122 \mathrm{~m}^{2} \cdot \mathrm{g}^{-1}$, so the experimental value for MCM-ACR is in accordance with our expectations. Table 1 summarizes the values obtained for the described MCM-41-sample as well as for both Sc(III)-free and Sc(III)-containing MCM-ACR.

The type IV BET isotherms of the material exhibit a large slope in the $\mathrm{N}_{2}$ uptake only at lower $p / p_{0}$ ratios, and they show no significant sorption hysteresis. We may therefore conclude that the silica obtained has a very uniform structure and a homogeneous composition, thus proving that the co-condensation could be carried out in a controllable way, and that the formation of larger mesopores was prevented in the presence of the organic precursor in spite of its rather bulky nature. 

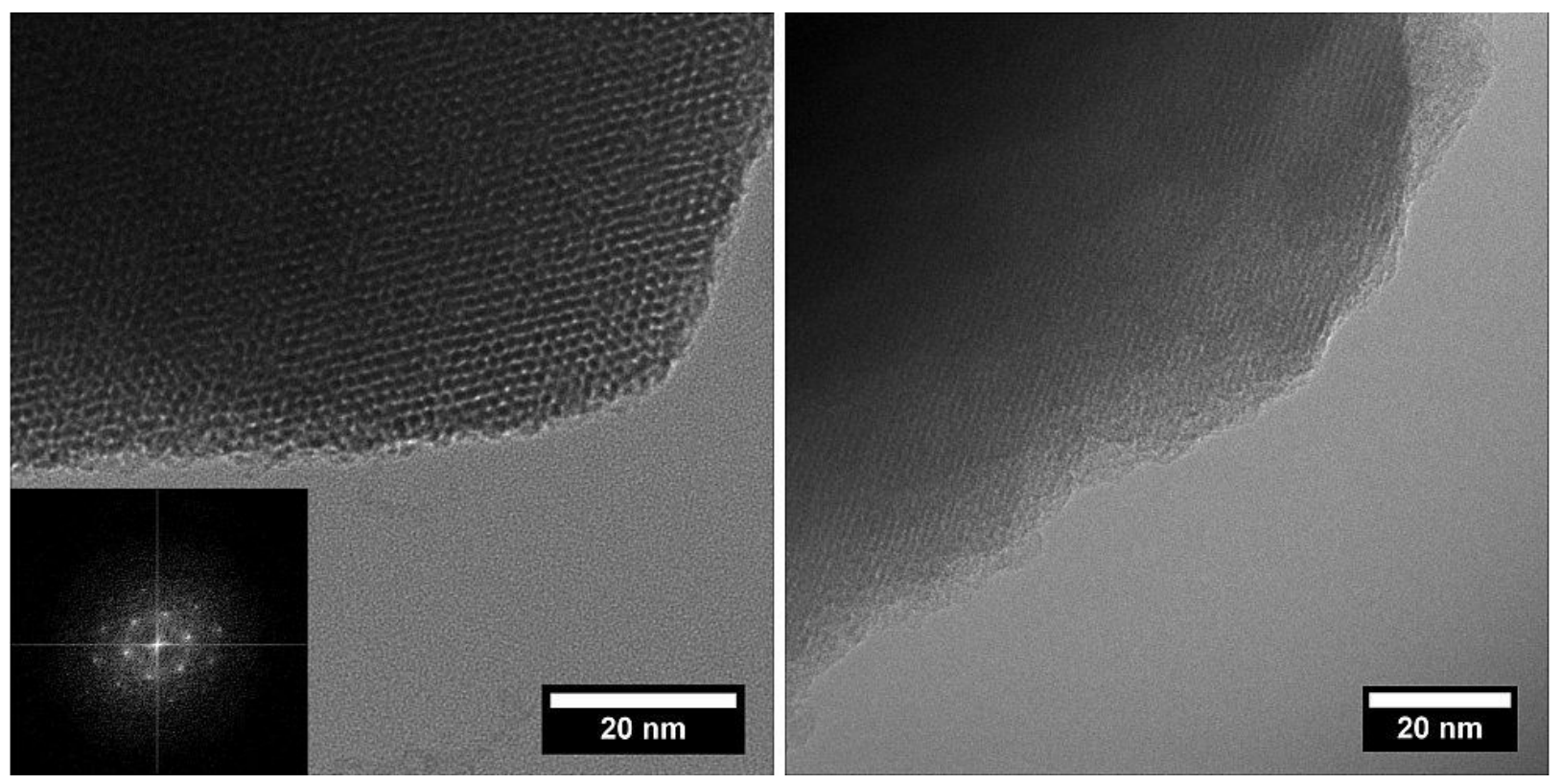

Figure 3: TEM images showing the mesoporous structure of MCM-ACR (left: frontal, right: lateral), inset in left image: Electron diffraction pattern.
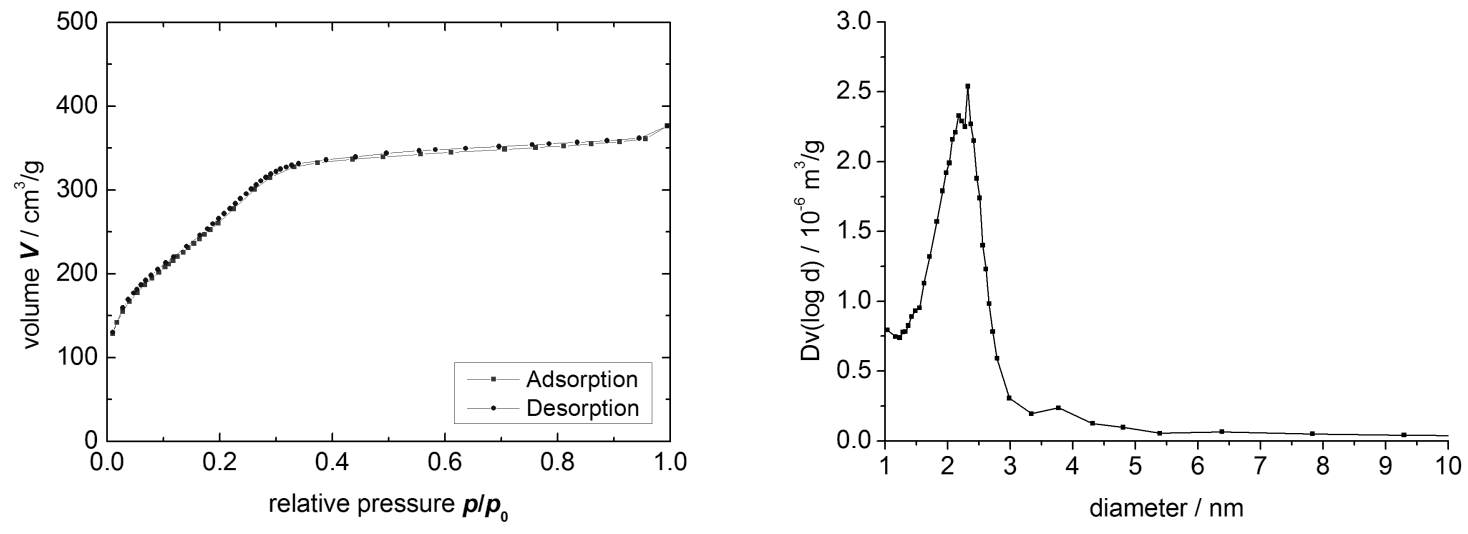

Figure 4: Sorption isotherm (left) and pore size distribution (BJH plot) (right) of MCM-ACR.

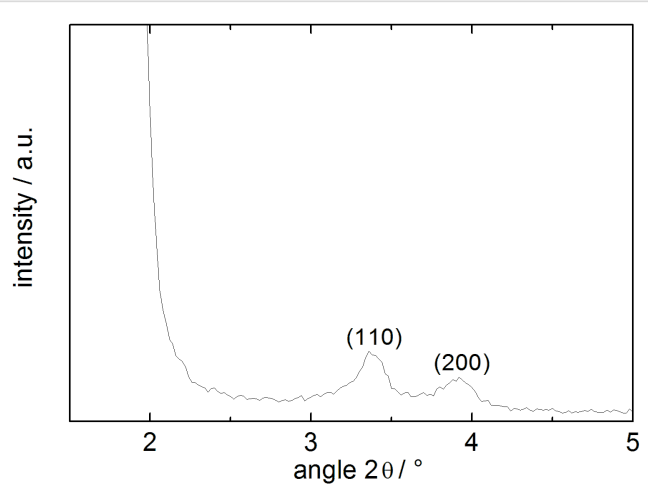

Figure 5: XRD pattern of MCM-ACR.
The ${ }^{13} \mathrm{C}$ CP-MAS NMR spectrum of MCM-ACR (Figure 6) shows the expected signals for the three methylene groups of the propyl chain as well as for the acridone moieties. Minor impurities in the spectrum of the precursor are mostly due to hydrolysis occurring during the measurement. The two prominent sharp alkyl peaks in the solid state spectrum may be attributed to the remaining free EtOH within the silicate. The ${ }^{29} \mathrm{Si}$ CP-MAS NMR data of MCM-ACR (Figure 7) prove that the material features the expected distribution of T- and Q-peaks. The minor $\mathrm{T}_{2}$-peak at $-57 \mathrm{ppm}$ can be ascribed to the $\mathrm{R}_{\text {alkyl }} \mathrm{Si}(\mathrm{OMe})(\mathrm{OSi})_{2}$ unit resulting from the partially incomplete incorporation of the precursor's anchoring group into the framework [20]. 
Table 1: BET and PSD date of different obtained mesoporous siliceous materials. In the case of MCM-ACR, two batches have been prepared with well reproducible outcomes (second batch in parentheses). The modified MCM-ACR sample was prepared from the first batch with a BET surface area of $775 \mathrm{~m}^{2} \cdot \mathrm{g}^{-1}$.

\begin{tabular}{cccc} 
type of material & BET surface $\left[\mathrm{m}^{2} \cdot \mathrm{g}^{-1}\right]$ & Langmuir surface $\left[\mathrm{m}^{2} \cdot \mathrm{g}^{-1}\right]$ & average pore diameter $[\mathrm{nm}]$ \\
\hline MCM-41 & 1122 & 2346 & 2.4 \\
MCM-ACR & $775(810)$ & $1135(1661)$ & 2.4 \\
MCM-ACR + Sc(OTf) 3 & 734 & 962 & 2.3
\end{tabular}

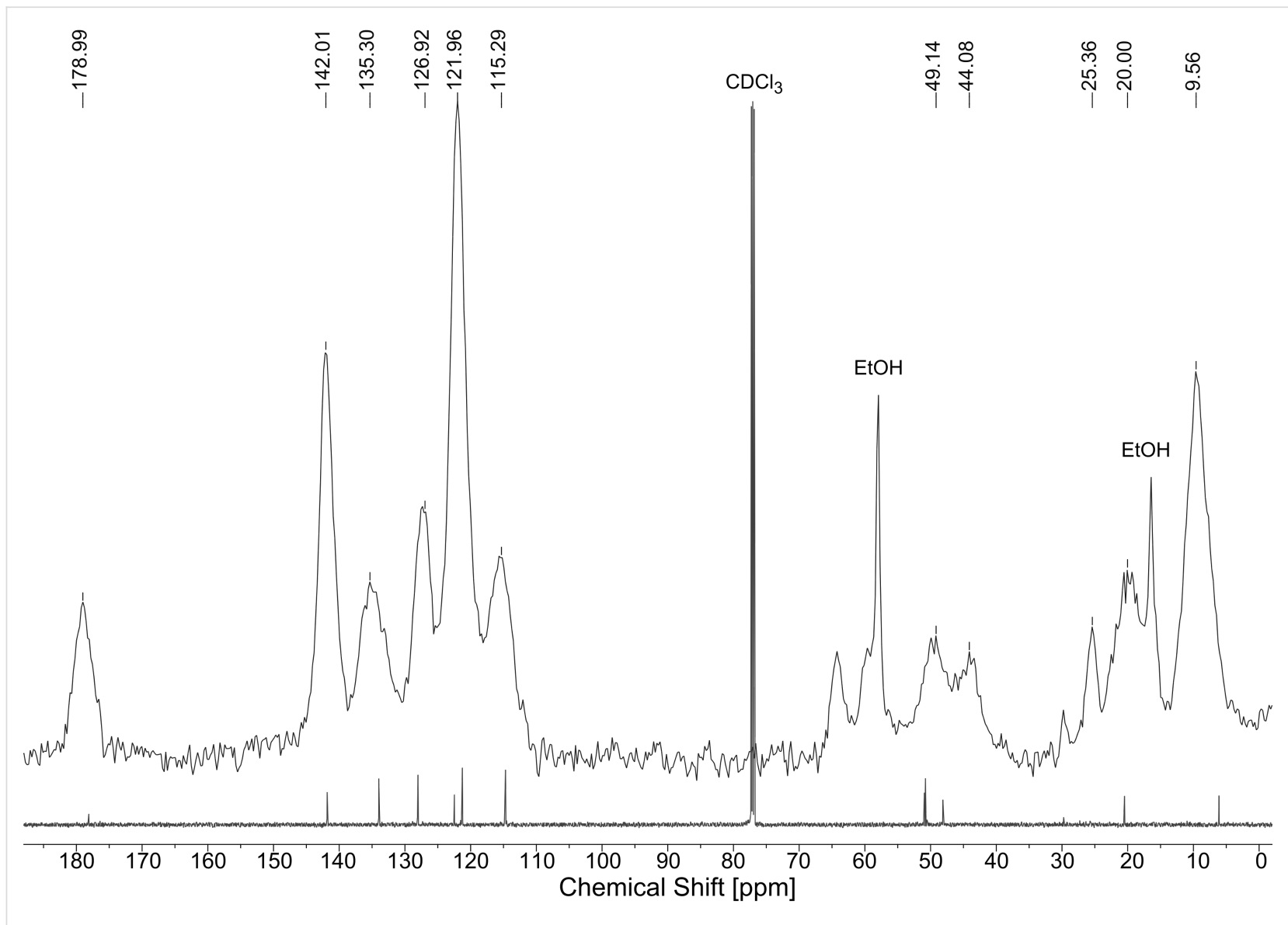

Figure 6: ${ }^{13} \mathrm{C}$ CP-MAS NMR spectrum of $\mathrm{MCM}-\mathrm{ACR}$ overlaid with the high resolution ${ }^{13} \mathrm{C}$ NMR spectrum of precursor 1 (recorded in $\mathrm{CDCl}_{3}$ ).

When studying the fluorescence properties of the materials obtained before and after the post-sol-gel modification, an instantaneous shift in color and fluorescence was observed when MCM-ACR and the scandium(III) triflate solution were combined. Compound 1, as well as unmodified MCM-ACR, feature a clear blue fluorescence when excited at a wavelength of $\lambda_{\text {ex. }}=366 \mathrm{~nm}$, whereas the complex formation with the scandium cation results in an intensely yellow colored product showing a greenish fluorescence (Figure 8). This effect is also clearly evident in the UV-vis and fluorescence spectra (Figure 9). Introduction of scandium(III) into the material yields a bathochromic effect in $\lambda_{\text {abs. }}$ and $\lambda_{\text {em. }}$ of about $20 \mathrm{~nm}$ and, more interestingly, a significantly increased absorption at around $\lambda_{\text {abs. }}=328 \mathrm{~nm}$. The appearance of this distinct absorption may hint towards an electron transfer (LMCT) from the molecular orbital located at the carbonyl oxygen to the empty $3 \mathrm{~d}$ orbital residing at the $\mathrm{Sc}(\mathrm{III})$ cation, a process which would be similar to those that have been described for carboxylate complexes of $\mathrm{Eu}(\mathrm{III})$ [21]. The fluorescence spectrum of the modified silica shows a distinct shoulder at around $\lambda_{\mathrm{em}} \approx 515 \mathrm{~nm}$. This indicates a complex radiative relaxation of the formed transition metal species, the fluorescence originating from at least two different transitions between the excited electronic states and the ground state. A reason for this 


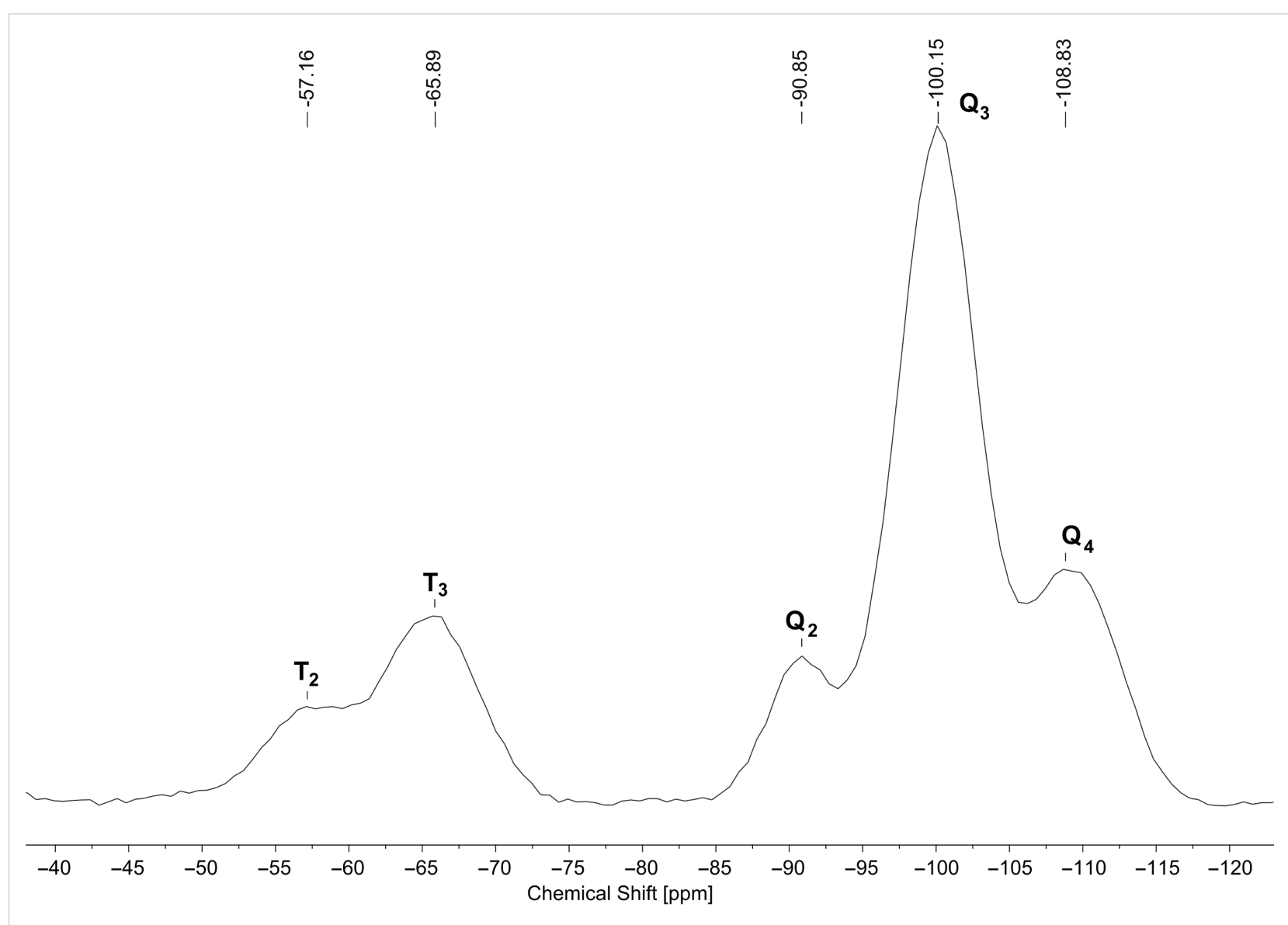

Figure 7: ${ }^{29}$ Si CP-MAS NMR spectrum of MCM-ACR.

phenomenon might be the energy splitting caused by the coupled CO stretching within the acridone- $\mathrm{Sc}^{3+}$ complex [7]. The pure triflate is almost completely transparent in the UV-vis region and shows no fluorescence when measured both as a solid film and in solution, hence it is obvious that the optical properties of the material depend on the electronic structure of the acridone chromophore, the latter being significantly changed via formation of the complex as the scandium(III) ion strongly interacts with the oxygen atom of the $\mathrm{C}=\mathrm{O}$ functional group. The complex itself seems to be very stable and inert to ligand exchange: Excessive washing with polar organic solvents and storing of an authentic sample for weeks, and even months, neither altered the appearance of the material nor decreased its fluorescence intensity.

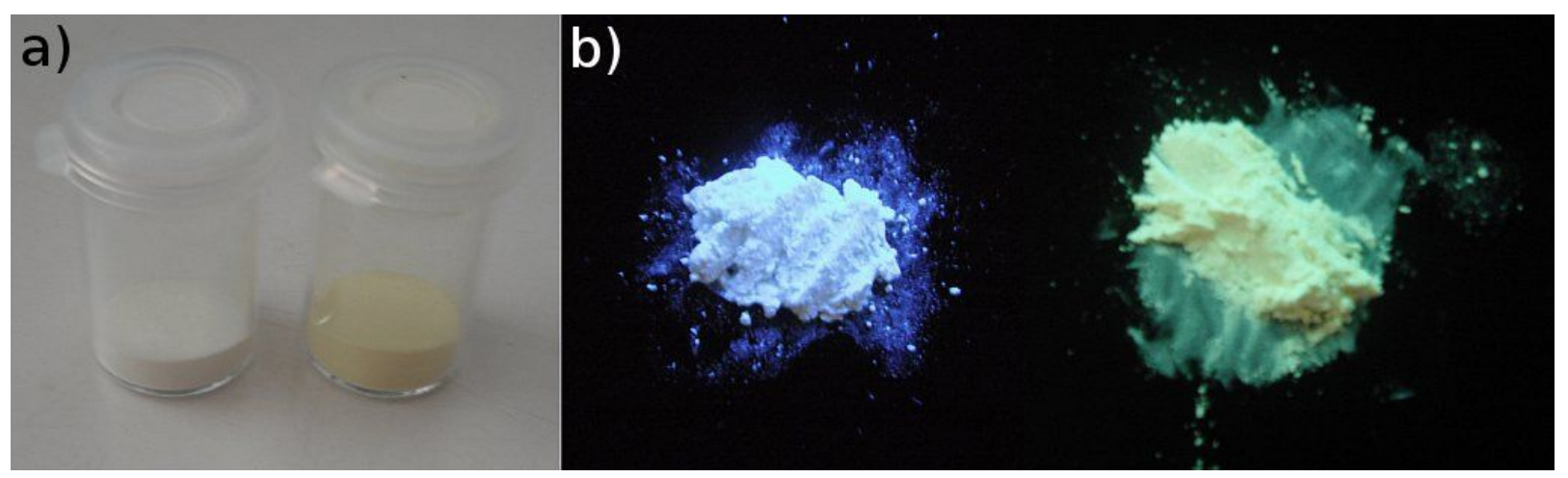

Figure 8: Visual appearance of MCM-ACR and MCM-ACR + Sc(OTf $)_{3}$ under normal (a) and UV light (b). 

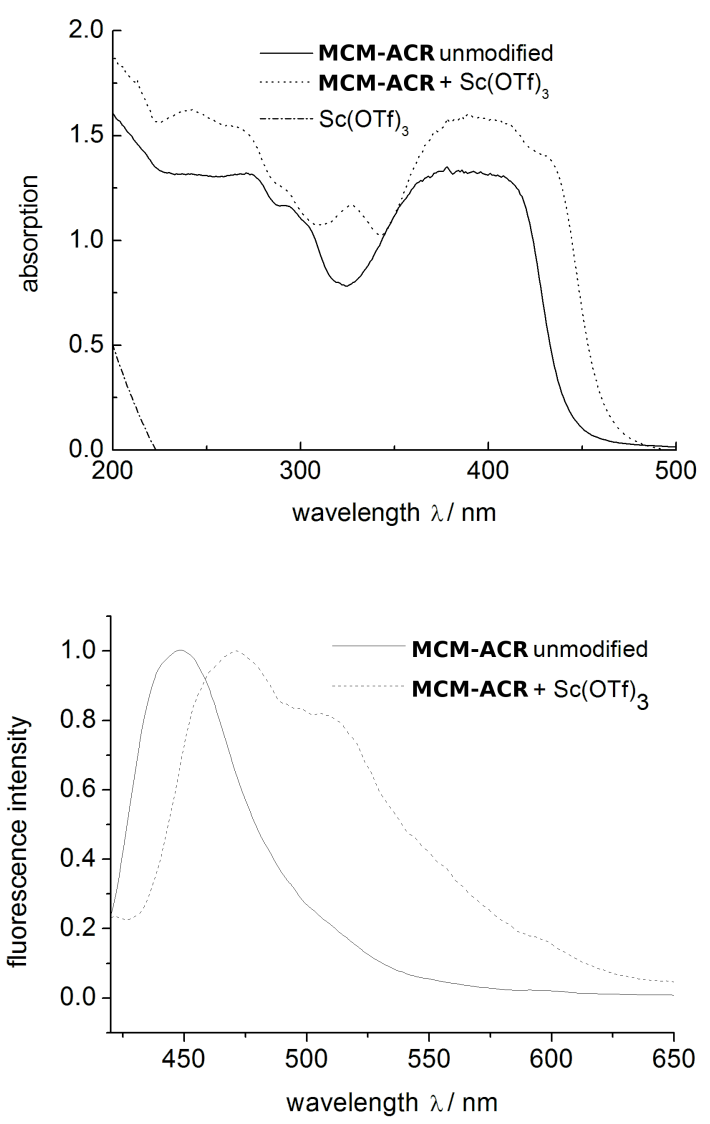

Figure 9: Overlay of the solid state UV-vis (top) and fluorescence (bottom) spectra of MCM-ACR, MCM-ACR + Sc(OTf $)_{3}$ and pure $\mathrm{Sc}(\mathrm{OTf})_{3}$ (fluorescence excitation wavelength $\left.\lambda_{\mathrm{ex}}=400 \mathrm{~nm}\right)$. UV-visabsorption intensities are not normalized to a given value; fluorescence has been normalized to an intensity of $1.0 . \mathrm{Sc}(\mathrm{OTf})_{3}$ shows no significant fluorescence and therefore was omitted in the second diagram.

\section{Conclusion}

We have presented a novel fluorescent organosilane bearing an acridone fluorophore and its successful transformation into a MCM-41 type material via co-condensation with TEOS. As predicted, the hybrid material shows a change in its fluorescence properties when non-covalently modified through scandium complex formation. The miscibility of the organic precursor or its concentrated solution with an excess of the major silicon source has been determined to be crucial for the synthesis of the hybrid material given herein. Possibilities to vary the dye content by using different molar ratios of the precursors in the sol-gel process are to be investigated in the near future. Furthermore, efforts will be made to elucidate the optical properties of the materials after doping with lanthanides or heavy metal cations, e.g. Eu(III), Er(III) or Bi(III).

\section{Experimental}

General: All starting materials described herein were purchased from Sigma-Aldrich and used as received. Solvents for the organic syntheses were dried prior to use according to standard procedures [22]. Solid-state ${ }^{1} \mathrm{H} \mathrm{CP}-\mathrm{MAS},{ }^{29} \mathrm{Si}$ CP-MAS and ${ }^{13} \mathrm{C}$ CP-MAS NMR spectra were recorded on a Bruker DSX Avance NMR spectrometer at resonance frequencies of $400 \mathrm{MHz}, 101 \mathrm{MHz}$ and $80 \mathrm{MHz}$ for ${ }^{1} \mathrm{H},{ }^{13} \mathrm{C}$ or ${ }^{29} \mathrm{Si}$ nuclei, respectively. Liquid phase ${ }^{1} \mathrm{H}$ and ${ }^{13} \mathrm{C}$ NMR spectra were recorded on Bruker Spectrospin DPX-400 and Avance 600 devices at resonance frequencies of $400 \mathrm{MHz}$ or $151 \mathrm{MHz}$ for ${ }^{1} \mathrm{H}$ or ${ }^{13} \mathrm{C}$ nuclei, respectively. These spectra are internally referenced to $\mathrm{SiMe}_{4}$. The infrared spectra with a resolution of $\pm 2 \mathrm{~cm}^{-1}$ were recorded using a PerkinElmer FT-ATR-IR 1000 spectrometer containing a diamond coated $\mathrm{ZnSe}$-window. MALDI-ToF measurements were conducted on a Bruker Daltonics Ultraflex spectrometer. Elemental analyses were determined on a CHNS vario Microcube elemental analyzer (Elementar). X-ray powder diffraction (PXRD) patterns of the silica samples were recorded on a Siemens D5005 instrument using Ni-filtered $\mathrm{Cu} \mathrm{K} \alpha$ radiation $(\lambda=1.5404 \AA)$, with a step size of $1 \% / \mathrm{min}$. $\mathrm{N}_{2}$-Adsorption-desorption isotherms, pore size distributions as well as the textural properties of the hybrid materials were determined at $77 \mathrm{~K}$ by a Quantachrome Autosorb 1 sorption analyzer. Before analysis, the samples were activated at $120{ }^{\circ} \mathrm{C}$ overnight in the vacuum and then the adsorption-desorption procedure was conducted by passing nitrogen into the sample, which was kept under liquid nitrogen. The average pore size of the samples was estimated using the $\mathrm{BJH}$ approach based on the Kelvin equation while assuming a cylindrically shaped porous structure. The specific surface areas were calculated by means of the Brunauer-Emmett-Teller (BET) equation in the low relative pressure interval $(<0.3)$ and the pore size distribution curves were analyzed with the adsorption branch by the BJH method. The morphology of the mesoporous particles was determined by a Philips CM 300 UT field emission transmission electron microscope (TEM) with $300 \mathrm{kV}$ acceleration voltage and $0.17 \mathrm{~nm}$ point resolution. The UV-vis absorption and fluorescence of the precursor were measured using a Perkin-Elmer Lambda 900 and a Horiba Jobin-Yvon Fluorolog 3-22 $\tau$ in steps of $0.1 \mathrm{~nm}$ and $1.0 \mathrm{~nm}$, respectively, the $\mathrm{SiO}_{2}$-cuvettes used had a width of $1.0 \mathrm{~cm}$. Solid state UV-vis measurements were carried out on a Perkin-Elmer Lambda 18 double beam UV-vis spectrometer with double monochromator by setting the wavelength range from $200 \mathrm{~nm}$ to $900 \mathrm{~nm}$ in a $1 \mathrm{~nm}$ step width. The optical unit included a prealigned tungsten-halogen lamp and a deuterium lamp with automatic source exchange. All powder samples were calibrated with a diffuse $\mathrm{BaSO}_{4}$ referenced auto zero and were measured using a Biconical (Praying Mantis) Diffuse Reflectance Accessory in reflecting absorption mode. Solid state fluorescence data 
was recorded on a Perkin-Elmer LS55 with a step width of $0.5 \mathrm{~nm}$. The thin film powder samples were prepared from a DCM suspension or solution by evaporation of the solvent on a glass substrate.

10-(Trimethoxysilylpropyl)acridin-9(10H)-one (1): Finely powdered dry acridone [23] (5.85 g, $30.0 \mathrm{mmol})$ was added portionwise to a stirred suspension of 1.1 equiv of sodium hydride $(800 \mathrm{mg}, 33.3 \mathrm{mmol})$ in dry THF $(100 \mathrm{~mL})$. The resulting yellow-greenish suspension was stirred for $15 \mathrm{~min}$ at $25^{\circ} \mathrm{C}$ until the hydrogen evolution subsided. Subsequently, 1.1 equiv of 3 -iodopropyltrimethoxysilane $(9.67 \mathrm{~g}, 6.53 \mathrm{~mL}$, $33.3 \mathrm{mmol}$ ) were added dropwise via a syringe through a rubber septum. The golden colored suspension was heated to reflux for $24 \mathrm{~h}$ before evaporation of the solvent. The residue was washed with dry pentane $(3 \times 10 \mathrm{~mL})$, then re-dissolved in several portions of dichloromethane (total of $150 \mathrm{~mL}$ ) and the combined organic solutions were filtered. Evaporation of the solvent gave 1 as a highly viscous orange oil (16.9 mmol, 6.77 g, 56\%) showing a strong blue-green fluorescence under UV light. ${ }^{1} \mathrm{H}$ NMR $\left(400 \mathrm{MHz}, \mathrm{CDCl}_{3}\right) \delta 8.61\left(\mathrm{~d},{ }^{3} J_{\mathrm{HH}}=7.9 \mathrm{~Hz}\right.$, $2 \mathrm{H}), 7.76\left(\mathrm{t},{ }^{3} J_{\mathrm{HH}}=7.8 \mathrm{~Hz}, 2 \mathrm{H}\right), 7.59\left(\mathrm{~d},{ }^{3} J_{\mathrm{HH}}=8.7 \mathrm{~Hz}, 2 \mathrm{H}\right)$, $7.32\left(\mathrm{t},{ }^{3} J_{\mathrm{HH}}=7.5 \mathrm{~Hz}, 2 \mathrm{H}\right), 4.51-4.34(\mathrm{~m}, 2 \mathrm{H}), 3.67(\mathrm{~s}, 9 \mathrm{H})$, $2.22-2.01(\mathrm{~m}, 2 \mathrm{H}), 0.89\left(\mathrm{t},{ }^{3} \mathrm{~J}_{\mathrm{HH}}=7.7 \mathrm{~Hz}, 2 \mathrm{H}\right) ;{ }^{13} \mathrm{C}$ NMR $(151$ $\left.\mathrm{MHz}, \mathrm{CDCl}_{3}\right) \delta 178.0,141.8,133.9,127.9,122.4,122.2,114.6$, 50.8, 48.1, 20.4, 6.1; ATR-IR (ZnSe) $\tilde{v}\left[\mathrm{~cm}^{-1}\right]: 2940,2840$, $1635,1599,1491,1462,1378,1342,1291,1264,1169,1045$, 956, 937, 897, 810, 753, 673; MALDI-ToF for $\mathrm{C}_{19} \mathrm{H}_{23} \mathrm{NO}_{4} \mathrm{Si}$ (matrix CHCA, $\left.\mathrm{M}^{+}\right)$: 356.6; UV-vis $\left(\mathrm{CH}_{2} \mathrm{Cl}_{2}, c \approx 10^{-6} \mathrm{M}\right) \lambda_{\text {abs. }}$. $=254,381,400 \mathrm{~nm}$; Fluorescence $\left(\mathrm{CH}_{2} \mathrm{Cl}_{2}, c \approx 10^{-6} \mathrm{M}, \lambda_{\text {ex. }}=\right.$ $250 \mathrm{~nm}): \lambda_{\mathrm{em} .}=415,431 \mathrm{~nm}$.

Acridone functionalized hybrid silica material (MCMACR): The molar ratio of the different sol-gel components was determined in advance to be 9.0:1.0:24.0:1.4:1000 (TEOS:precursor 1:base: $\mathrm{C}_{18} \mathrm{TAB}: \mathrm{H}_{2} \mathrm{O}$ ). In order to achieve a homogeneous co-condensation, the organic precursor ideally had to be dissolved prior to hydrolysis. An aqueous $70 \mathrm{wt} \%$ solution of $\mathrm{H}_{2} \mathrm{NEt}(12.2 \mathrm{~g}, 190 \mathrm{mmol})$ was introduced into a stirred solution of $\mathrm{C}_{18} \mathrm{TAB}(4.35 \mathrm{~g}, 11.1 \mathrm{mmol})$ in deionized water $(142 \mathrm{~mL})$. A two-phase mixture consisting of TEOS (14.8 g, $15.7 \mathrm{~mL}, 71.0 \mathrm{mmol})$ and $\mathbf{1}(3.15 \mathrm{~g}, 8.82 \mathrm{mmol})$ was rapidly added at $25^{\circ} \mathrm{C}$ by syringe under vigorous stirring. The milky yellow solution, which soon contained a precipitate, was stirred at room temperature for $5 \mathrm{~h}$ before being heated to $100-110^{\circ} \mathrm{C}$ (bath temperature) for a further $16 \mathrm{~h}$. The resulting hot suspension was filtered, washed thoroughly with deionized water (a total amount of $1 \mathrm{~L}$ ) and the solid residue was re-suspended in a 1:8 mixture of ethanol and concentrated $\mathrm{HCl}$ (200 mL). $\mathrm{C}_{18} \mathrm{TAB}$ was extracted by stirring for $16 \mathrm{~h}$ at $85^{\circ} \mathrm{C}$. The obtained solid was filtered from the hot solution, washed with $\mathrm{EtOH}(500 \mathrm{~mL})$ and dried in the vacuum to give the product as a pale yellow, very fine powder $(6.60 \mathrm{~g}, 7.76 \mathrm{mmol}$ according to a calculated molecular weight of $835.7 \mathrm{~g} \cdot \mathrm{mol}^{-1}$ ). CHNS analysis found: $\mathrm{C} 21.25, \mathrm{H} 3.56, \mathrm{~N} 1.66$; calcd. for $\left(\mathrm{C}_{16} \mathrm{H}_{14} \mathrm{NO}_{2.5} \mathrm{Si}\right) \cdot\left(\mathrm{H}_{2} \mathrm{O}\right)_{9} \cdot\left(\mathrm{SiO}_{2}\right)_{7.5}$ : C $21.33, \mathrm{H} 3.48, \mathrm{~N} 1.55$. This gives a $\mathrm{CHN}$ content of approx. $24 \mathrm{wt} \%$ (related to the $N$-propylacridone moiety).

Post-sol-gel modification of MCM-ACR: Introduction of the scandium salt into the material was realized by stirring MCMACR (500 mg) in a $0.01 \mathrm{M}$ solution of scandium(III) triflate in ethanol or acetonitrile $(25 \mathrm{~mL})$ for $16 \mathrm{~h}$. The modified materials were thoroughly washed with ethanol or acetonitrile $(5 \times$ $5 \mathrm{~mL})$ and water $(5 \times 5 \mathrm{~mL})$ prior to drying and characterization.

\section{Acknowledgements}

We gratefully thank the Konrad-Adenauer-Stiftung (KAS) for funding the Ph. D. thesis of M. H. and the Carl-Zeiss-Stiftung for the financial support of Y. S. .

\section{References}

1. Wang, X.; Chen, C.-C.; Chen, S.-Y.; Mou, Y.; Cheng, S. Appl. Catal., A 2005, 281, 47-54. doi:10.1016/j.apcata.2004.11.011

2. Hu, Y.; Higashimoto, S.; Martra, G.; Zhang, J.; Matsuoka, M.; Coluccia, S.; Anpo, M. Catal. Lett. 2003, 90, 161-163. doi:10.1023/B:CATL.0000004111.02392.75

3. Corma, A. Chem. Rev. 1997, 97, 2373-2420. doi:10.1021/cr960406n

4. Zhou, Z.; Franz, A. W.; Hartmann, M.; Seifert, A.; Müller, T. J. J.; Thiel, W. R. Chem. Mater. 2008, 20, 4986-4992. doi: $10.1021 / \mathrm{cm} 800804 \mathrm{t}$

5. Franz, A. W.; Zhou, Z.; Turdean, R.; Wagener, A.; Sarkar, B.; Hartmann, M.; Ernst, S.; Thiel, W. R.; Müller, T. J. J. Eur. J. Org. Chem. 2009, 3895-3905. doi:10.1002/ejoc.200900332

6. Zhou, Z.; Franz, A. W.; Bay, S.; Sarkar, B.; Seifert, A.; Yang, P.; Wagener, A.; Ernst, S.; Pagels, M.; Müller, T. J. J.; Thiel, W. R. Chem.-Asian J. 2010, 5, 2001-2015. doi:10.1002/asia.201000098

7. Bahr, N.; Tierney, E.; Reymond, J.-L. Tetrahedron Lett. 1997, 38, 1489-1492. doi:10.1016/S0040-4039(97)00137-8

8. Fukuzumi, S.; Ohkubo, K. J. Am. Chem. Soc. 2002, 124, 10270-10271. doi:10.1021/ja026613o

9. Cejas, M. A.; Raymo, F. M. Langmuir 2005, 21, 5795-5802. doi:10.1021/la0502793

10. Sambhy, V.; Peterson, B. R.; Sen, A. Langmuir 2008, 24, 7549-7558. doi:10.1021/la800858z

11. Biazzotto, J. C.; Sacco, H. C.; Ciu, K. J.; Neri, C. R.; Ferreira, A. G.; lamamoto, Y.; Serra, O. A. J. Non-Cryst. Solids 1999, 247, 134-140. doi:10.1016/S0022-3093(99)00050-2

12. Schneider, M.; Müllen, K. Chem. Mater. 2000, 12, 352-362. doi:10.1021/cm9910613

13. Lam, M. H. W.; Lee, D. Y. K.; Man, K. W.; Lau, C. S. W. J. Mater. Chem. 2000, 10, 1825-1828. doi:10.1039/b001914n

14. Lin, Y.-C.; Chen, C.-T. Org. Lett. 2009, 11, 4858-4861. doi:10.1021/ol901935g

15. Lin, W.; Cai, Q.; Pang, W.; Yue, Y. Chem. Commun. 1998, 2473-2474. doi:10.1039/a807786j 
16. Furukawa, H.; Takeuchi, S.; Moriguchi, I.; Teraoka, Y.; Kagawa, S. Nippon Kagakkai Koen Yokoshu 1998, 75, 284. http://sciencelinks.jp/j-east/article/199907/000019990799A0248134.ph $\underline{p}$

17. Berezin, K. V.; Krivokhizhina, T. V.; Nechaev, V. V. Opt. Spectrosc. 2006, 100, 15-22. doi:10.1134/S0030400X0601005X

18. Spectral Database for Organic Compounds SDBS, National Institute of Advanced Industrial Science and Technology (AIST). http://www.aist.go.jp/ (accessed Feb 11, 2011).

19. Spange, S.; Zimmermann, Y.; Gräser, A. Chem. Mater. 1999, 11, 3245-3251. doi:10.1021/cm990308t

20. Bein, T.; Carver, R. F.; Farlee, R. D.; Stucky, G. D. J. Am. Chem. Soc. 1988, 110, 4546-4553. doi:10.1021/ja00222a010

21. An, B.-L.; Gong, M.-L.; Cheah, K.-W.; Zhang, J.-M.; Li, K.-F. Chem. Phys. Lett. 2004, 385, 345-350. doi:10.1016/j.cplett.2003.12.093

22. Riddick, J. A.; Bunger, W. B.; Sakano, T. K. Organic Solvents: Physical Properties and Methods of Purification, 4th ed.; Wiley: New York, 1986. 23. Allen, C. F. H.; McKee, G. H. W. Organic Syntheses, Coll. Vol. 2 1943, 15.

http://www.orgsyn.org/orgsyn/prep.asp?prep=cv2p0015

\section{License and Terms}

This is an Open Access article under the terms of the Creative Commons Attribution License

(http://creativecommons.org/licenses/by/2.0), which permits unrestricted use, distribution, and reproduction in any medium, provided the original work is properly cited.

The license is subject to the Beilstein Journal of

Nanotechnology terms and conditions:

(http://www.beilstein-journals.org/bjnano)

The definitive version of this article is the electronic one which can be found at:

doi:10.3762/bjnano.2.33 